\title{
Comparative study of antioxidant activity of some amides
}

\begin{abstract}
The aim of this study was to evaluate the antioxidant activities of five amides: benzanilide 1, dodecanilide 2, $\mathrm{N}$-cyclohexyloctamide 3 , acetanilide 4, and acetaminophen (paracetamol) 5 , and to compare them to those of standard antioxidants, i.e. butylated hydroxyanisole (BHA), butylated hydroxy toluene (BHT), ascorbic acid (vitamin C) and $\alpha$-tocopherol (vitamin E). Three common experimental tests were used to assess their antioxidant properties: 2,2-diphenyl-1-picrylhydrazyl (DPPH) radical scavenging activity, ferric ions reducing antioxidant power (FRAP) and $\beta$-carotene/linoleic acid assays. The amides 1-3 proved to be antioxidant as per three methods; the fatty anilide 2 showed the highest radical scavenging activity, whereas the fatty amide 3 showed the lowest one. Increasing the concentration resulted in an increased ferric reducing antioxidant power for all the examined amides; the reducing power of fatty anilide 2 was relatively higher than those of benzanilide 1 and fatty amide 3. Oxidation of the linoleic acid was strongly inhibited by all amides. The obtained results were comparable to antioxidant properties of the standard antioxidants.
\end{abstract}

Keywords: amide derivatives, antioxidant activity, $\beta$-Carotene bleaching, DPPH assay, nFRAP assay
Volume 5 Issue 3 - 2017

\author{
Fatiha Malki,' Abdelkader Touati,' Saâd \\ Moulay ${ }^{2}$ \\ 'Laboratoire de recherche sur les produits bioactifs et \\ valorisation de la biomasse, Algeria \\ ${ }^{2}$ Département de Chimie Industrielle, Université Saâd Dahlab \\ de Blida,Algeria
}

Correspondence: Fatiha Malki, Laboratoire de recherche sur les produits bioactifs et valorisation de la biomasse, Ecole Normale Sup, Email malki_fatiha76@yahoo.fr

Received: June 01, 2017| Published: July 06, 2017
Abbreviations: BHA, Butylated Hydroxyanisole; BHT, Butylated Hydroxy Toluene; Vitamin C, Ascorbic Acid; Vitamin E, Tocopherol; DPPH, Diphenyl-1-Picrylhydrazyl; FRAP, Ferric Ions Reducing antioxidant power; $\mathrm{AD}$, alzheimer's disease; $\mathrm{PD}$, parkinson's disease; ALS, amyotrophic lateral sclerosis

\section{Introduction}

To recall, lipids containing carbon-carbon double bond(s) are vulnerable to oxidation under aerobic conditions and are easily attacked by free radicals, giving rise to harmful products; such deteriorating phenomenon is called 'lipid oxidation'. Antioxidants are organic molecules which can scavenge free radicals and thus avoid or delay the progress of this lipid oxidation. For this matter, they generally exist as additives in food products. They are promising agents for management of oxidative stress related diseases such as cancer, arthritis, aging, autoimmune disorders, Alzheimer's disease (AD), Parkinson's disease (PD) and amyotrophic lateral sclerosis (ALS). ${ }^{1}$ Therefore, there is a growing interest in substances that exhibit antioxidant properties, which are supplied to humans and animals as food components or as specific pharmaceuticals. Several methods have been developed to evaluate antioxidant activities of natural and synthetic substances., ${ }^{2,3}$

Amide derivatives play an important role in biological activities. ${ }^{4-7}$ For example, the uses of the class of anilides in medicine, pharmacy, biology and other related fields well-known and are as such because of their biological activities including anti-bacterial, anti-fungicidal, anti-convulsant, anesthetic, and platelet aggregation. ${ }^{10}$ Various anilides have also found a wide applicability as bioactive species (antimicrobial, antioxidant and antatherosclerotic agents). ${ }^{11,12}$ Moreover, they are also involved as intermediate products in the synthesis of therapeutic agents. ${ }^{8,9}$

In the present work, the antioxidant activities of three synthesized amides: benzanilide 1, dodecanilide 2, $\mathrm{N}$-cyclohexyloctamide 3 , and of two known ones: acetanilide 4 and acetaminophen (paracetamol) 5 , were evaluated and compared to those of standard antioxidants such as butylated hydroxyanisole (BHA), butylated hydroxy toluene (BHT), ascorbic acid (vitamin C) and $\alpha$-tocopherol (vitamin E). The antioxidant activities were estimated with three different methods: 2,2-diphenyl-1-picrylhydrazyl (DPPH) radical scavenging activity, ferric ions reducing antioxidant power (FRAP) and $\beta$-carotene/ linoleic acid assays.

\section{Materials and methods}

\section{Chemicals}

Chemicals were of analytical grade and were purchased from one of the following chemical companies: Sigma-Aldrich, Merck, Prolabo, and Biochem.

\section{Synthesis}

The three synthesized amides (1-3) were prepared from aniline and carboxylic acids by the reported procedures. ${ }^{13,14}$ The chemical structures of the tested amides are illustrated in Figure 1.

General synthetic procedure for 1, 2, and 3: Benzanilide 1 was first prepared via a solvent-free reaction by the conventional method as described earlier by ${ }^{14}$ as follows. A mixture of aniline and benzoic acid was heated at a temperature range of $160-200^{\circ} \mathrm{C}$ for a time period of $3-4 \mathrm{~h}$. The water from the reaction was continuously distilled off (Eq.1).

Under identical operating conditions, dodecanilide 2 was prepared by reaction of an excess of aniline with dodecanoic acid (Eq. 1) ${ }^{15}$ and $\mathrm{N}$-cyclohexyloctanamide 3 was obtained by reaction of an excess of cyclohexylamine with octanoic acid. The produced white solids were recrystallized in ethanol to afford colorless needle-like crystals. The different amides 1-3 were obtained as white crystals in yields as high as $73-92 \%$. 


\section{Antioxidant assay}

The antioxidant activity is the capability of a compound to inhibit the oxidative degradation, e.g. lipid peroxidation. ${ }^{16}$ In this study we have applied three tests to evaluate the potential antioxidant of the amides 1-5. Antioxidant activities of standard antioxidants (BHT, BHA, ascorbic acid (vitamin C) and $\alpha$-tocopherol (vitamin E) used as references were determined in parallel experiments.

DPPH radical scavenging assay: DPPH has been widely used for the evaluation of free radical scavenging effectiveness of various antioxidant substances. ${ }^{17}$ This method is based on the reduction of an alcoholic DPPH solution in the presence of a hydrogen-donating antioxidant. ${ }^{18}$ The capacities of amides to donate hydrogen atoms are measured from the discoloration of the purple methanolic solution of free radical 2, 2 diphenyl-1-picrylhydrazyl (DPPH). The free radical scavenging capacity of anilides was determined using the DPPH according to the method of Blois with some modifications. ${ }^{19}$ A solution of DPPH $(0.004 \%)$ in methanol was prepared and $1 \mathrm{~mL}$ of this solution was mixed with $1 \mathrm{~mL}$ of varying concentrations of amides solution in ethanol. The reaction mixture was vortexed thoroughly and left in the dark at room temperature for $3 \mathrm{~h}$. The absorbance of the mixture was measured at $\lambda_{\max }=517 \mathrm{~nm}$ using BHA, BHT, $\alpha$-tocopherol (vitamin E) and ascorbic acid (vitamin $\mathrm{C}$ ) as standards. The radical scavenging activity was calculated using the following formula:

\section{$D P P H$ radical scavenging activity $(\%)=[(A c-A s)] /(A c)] \mathrm{X} 100$}

Where AC is the absorbance of the control reaction (DPPH solution without the compound to be tested) and AS the absorbance of sample. The concentration of anilides providing 50\% inhibition $\left(\mathrm{IC}_{50}\right)$ was calculated from the graph of the plot of inhibition extent (in \%) against amide concentration (in $\mu \mathrm{g} / \mathrm{mL}$ ). ${ }^{20,21}$

Reducing power assay: The reducing power of a substance is a measure of its reductive ability as antioxidant, and it is estimated by the transformation of ferric ion $\mathrm{Fe}^{3+}$ to ferrous one $\mathrm{Fe}^{2+}$ in the presence of the sample extract. ${ }^{21}$ The ability to reduce $\mathrm{Fe}^{3+}$ can be attributed to the hydrogen donation capacity from phenolic compounds as described by Shimada et al..$^{22}$ In this assay, the yellow color of the test solution turns green or blue according to the reducing power of the test sample. The capacity of the antioxidant to reduce the ferric ferricyanide complex to the ferrous one was determined based on absorbance at $700 \mathrm{~nm}$ after incubation; the more intense the absorption, the greater the reducing power. ${ }^{23,24}$

The ferric reducing potential of anilides was assayed as described by Oyaisu. ${ }^{25}$ Different concentrations of amides $1-5$ in $1 \mathrm{~mL}$ of ethanol were mixed with $2.5 \mathrm{~mL}$ of phosphate buffer $(0.2 \mathrm{M}, \mathrm{pH} 6.6)$ and $2.5 \mathrm{~mL}$ of potassium ferricyanide $\left[\mathrm{K}_{3} \mathrm{Fe}(\mathrm{CN})_{6}\right](1 \%)$, and then the mixture was incubated at $50^{\circ} \mathrm{C}$ for $20 \mathrm{~min}$. Afterwards, $2.5 \mathrm{~mL}$ of trichloroacetic acid (10\%) was added to the mixture, which was then centrifuged at $1000 \mathrm{rpm}$ for $10 \mathrm{~min}$. Finally, $2.5 \mathrm{~mL}$ of the upper layer solution was mixed with $2.5 \mathrm{~mL}$ of distilled water and $0.5 \mathrm{~mL}$ of $\mathrm{FeCl}_{3}$ $(0.1 \%)$, and the absorbance was measured at $\lambda_{\max }=700 \mathrm{~nm}$.

\section{$\beta$-Carotene bleaching assay}

$\beta$-Carotene bleaching method is based on $\beta$-carotene oxidation (discoloring) induced by the products from linoleic acid oxidative degradation. The presence of an antioxidant prevents the reducing action of $\beta$-carotene which remains yellowish-orange in color. ${ }^{26}$

$\beta$-Carotene bleaching assay was conducted by using the method suggested by $\mathrm{Tepe}^{27}$ with some modifications. A solution of $\beta$-carotene was prepared by dissolving $0.5 \mathrm{mg}$ of $\beta$-carotene in $1 \mathrm{~mL}$ of chloroform. $25 \mu \mathrm{L}$ of linoleic acid and $200 \mathrm{mg}$ of Tween 80 were added. Chloroform was completely evaporated using a vacuum evaporator. Then, $100 \mathrm{~mL}$ of distilled water saturated with oxygen were added with a vigorous shaking. $2.5 \mathrm{~mL}$ of this reaction mixture were dispersed in test tubes and $350 \mu \mathrm{L}$ portions of the samples prepared at $2 \mathrm{mg} / \mathrm{mL}$ concentrations were added; the ensued emulsion system was incubated up to 48 hours at room temperature. The same procedure was repeated for positive control BHT and a blank. The absorbances of the mixtures were measured at $\lambda_{\max }=490 \mathrm{~nm}$ at regular time intervals. The relative antioxidant activity (AA) was calculated according to the following equation:

$$
A A \%=\left(A s_{(48)} / A c_{(48)}\right) \mathrm{X} 100
$$

Where $\mathrm{A}_{\mathrm{S}(48)}$ is the absorbance of the sample after $48 \mathrm{~h}$ and $\mathrm{A}_{\mathrm{C}(48)}$ the absorbance of BHT used as a positive control.

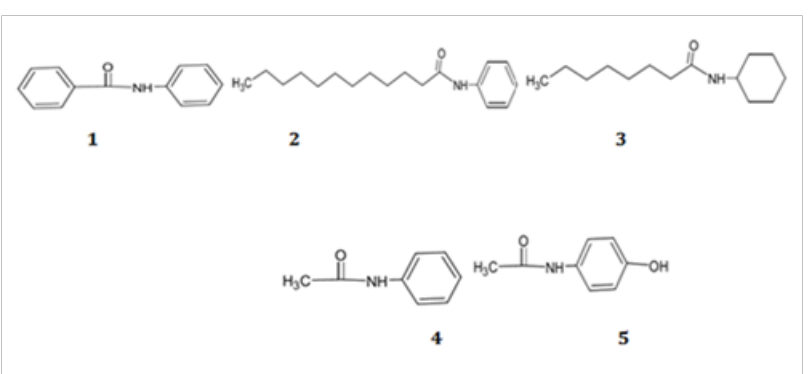

Figure I Chemical structures of the tested amides I-5.

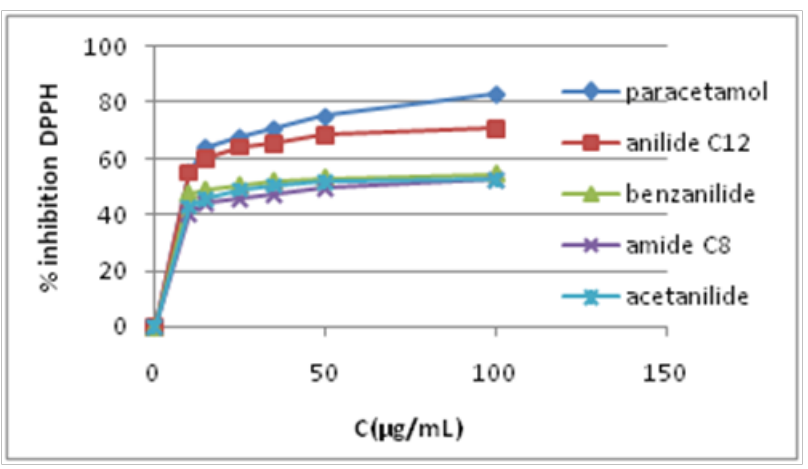

Figure 2 Radical scavenging activity of DPPH against amides I-5.

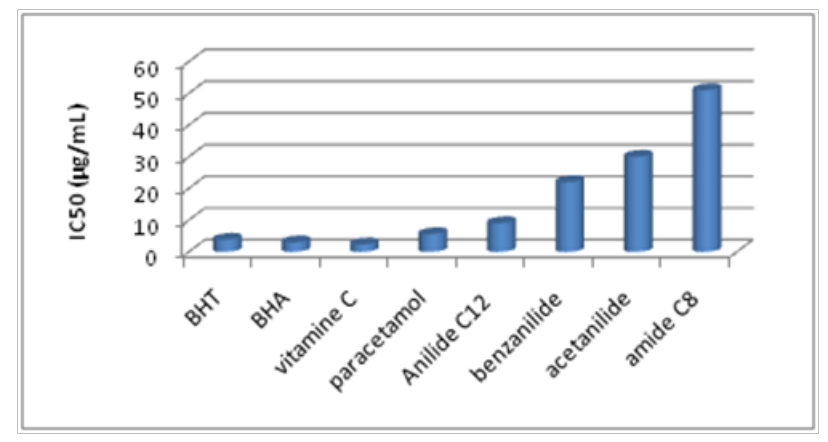

Figure $3 \mathrm{IC}_{50}$ values $(\mu \mathrm{g} / \mathrm{mL})$ of amides $\mathrm{I}-\mathbf{5}$ and reference antioxidants in the $\mathrm{DPPH}$ assay.

\section{Results and discussion}

\section{Synthesis}

The derivative anilides synthesized were obtained in quantitative yields. Their structures were confirmed by spectroscopic analyses, including UV-visible, IR, ${ }^{1} \mathrm{H}-\mathrm{NMR}$ and MS, as reported earlier. ${ }^{13}$ 


\section{Antioxidant activity}

DPPH radical scavenging activity: DPPH is a common reagent used to quantify the free radical scavenging activity of antioxidants. By virtue of being a stable free radical, the delocalization of the free electron gives rise to a deep violet color, characterized by an absorption band in ethanol solution at about $517 \mathrm{~nm}$.

In the DPPH radical-scavenging assay, an antioxidant reacts with DPPH, leading to the reduced form of the latter; DPPH pulls out either an electron or hydrogen atom from the antioxidant. As a result, the color changes from violet to yellow. The color fading extent proves indirectly the radical-scavenging capacity of the antioxidant. ${ }^{19}$

DPPH has been used extensively as a free radical to evaluate reducing substances ${ }^{28}$ and is a useful reagent for investigating the free radical scavenging activities of compounds. ${ }^{29}$ In its radical form, DPPH absorbs at $517 \mathrm{~nm}$, but upon reduction with an antioxidant, its absorption decreases due to the formation of its non-radical form, DPPH-H. ${ }^{4}$ Thus, the radical scavenging activity in the presence of a hydrogen donating antioxidant can be monitored as a decrease in absorbance of DPPH solution. ${ }^{3}$ The results of percentage inhibition of the DPPH radical by standard antioxidants and the different amides are represented in Figure 2.

As can be seen, the amides present a scavenging capacity of free radicals. It is also noted that the efficiency of the antioxidant increased with the concentration of the tested amides. $\mathrm{The} \mathrm{IC}_{50}$ values, which are concentrations of antioxidants required to reduce $50 \%$ the DPPH concentration, are illustrated in Figure 3.

As shown in Figure 3, all amides presented a scavenging capacity of free radicals. Anilide 5 (paracetamol) with a hydroxyl group exhibited higher activity, however, the amide 3 (amide C8), was the least active. Based on the $\mathrm{IC}_{50}$ values, the antioxidant capacity of the tested amides was lower than that of the control molecules. The DPPH scavenging potential of anilides may be due to their reducing action by donating hydrogen atom to a free radical, reducing it to nonreactive species. ${ }^{30}$

\section{Reducing power assay}

The reducing power of a compound is related to its transfer capacity of electrons and can serve as a significant indicator of its potential antioxidant activity. ${ }^{21}$ The reducing powers of standard antioxidants and of synthesized anilides at $700 \mathrm{~nm}(\mathrm{C}=100 \mu \mathrm{g} / \mathrm{mL})$ are presented in Figure 4.

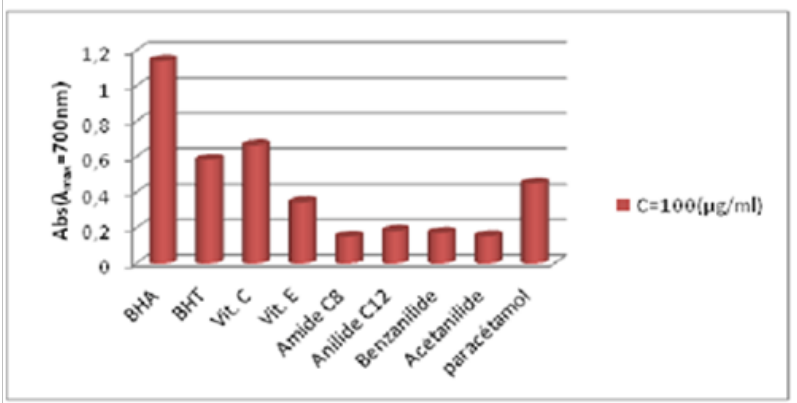

Figure 4 Reducing power of anilides I-5 and reference antioxidants at $700 \mathrm{~nm}$. $(\mathrm{C}=100 \mu \mathrm{g} / \mathrm{mL})$.

As can be seen in Figure 4, the amides 1-5 demonstrated powerful $\mathrm{Fe}^{3+}$ reducing ability. By comparing the obtained results, we note that the anilide 5 (paracetamol) is the most active, whereas a weaker reducing power was observed for the amide 3 . It can be noticed that the reducing powers of these compounds were close to that of vitamin $\mathrm{E}$ but were lower than that of the remaining reference antioxidants (BHT, BHA and vitamin C).

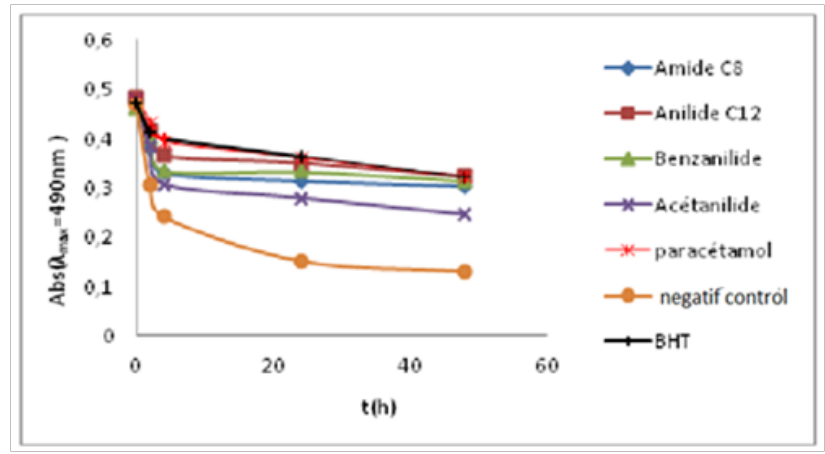

Figure 5 Antioxidative potentials of amids (I-5) and positive control BHT in $\beta$-carotene/linoleic acid system.

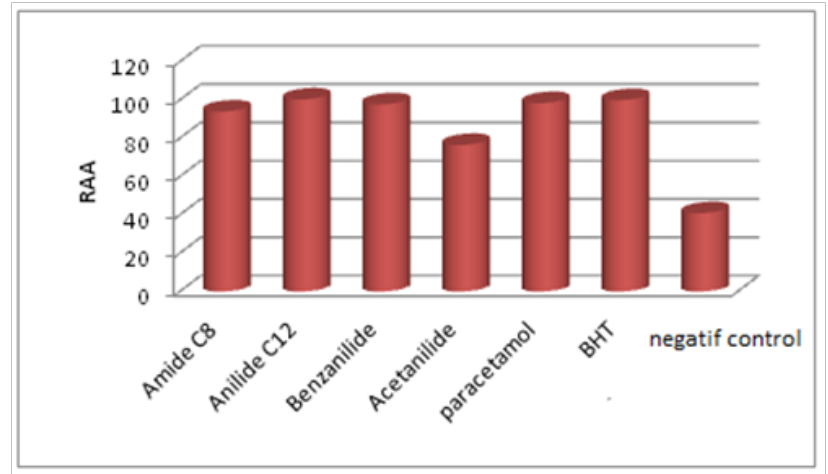

Figure 6 Relative antioxidant activity (RAA) of various amides and positive control BHT in $\beta$-carotene/linoleic acid system.

\section{$\beta$-Carotene bleaching assay}

The $\beta$-carotene/linoleic acid test determines the inhibition rate of the oxidation of linoleic acid to confirm anti-lipoperoxidation effects of sample. The $\beta$-carotene bleaching method is widely used to measure the antioxidant activity of plant extracts. This method is based on the fact that linoleic acid produces a free radical which is reduced by $\beta$-carotene. The addition of an antioxidant allows a delay of the discoloration kinetics of $\beta$-carotene. ${ }^{26}$ According to several authors, the test of inhibition of the oxidation of linoleic acid coupled with $\beta$-carotene, appeared very useful as a model of lipid peroxidation in biological membranes. ${ }^{32}$

The bleaching kinetics of $\beta$-carotene in the presence of the synthesized amides and reference antioxidants (BHT) are shown in Figure 5. As seen in Figure 5, all anilides inhibited the oxidation of $\beta$-carotene. This effect is due to either the inhibition of linoleic acid peroxidation or the radical scavenging of hydroperoxides formed during the peroxidation of linoleic acid (scavenger effect). The antioxidant activity (RAA) of anilides relative to that of the BHT in $\beta$-carotene/linoleic acid system is shown in Figure 6. The results in Figure 6 would suggest that all the amides inhibited efficiently the oxidation of linoleic acid $/ \beta$-carotene system when compared to the negative control. These values were very similar to that of the antioxidant BHT.

\section{Conclusion}

In this work, we evaluated the antioxidant activity of five amides through three methods: 2, 2-diphenyl-1-picryl-hydrazil (DPPH) free radical scavenging, Ferric reducing antioxidant power (FRAP), and 
bleaching of $\beta$-carotene. The results showed that the amides exhibit considerable antioxidant activities compared to those of the reference antioxidants. The antioxidant efficacy increases with the concentration of anilides and is directly related to the nature of the substituents on the amide group. These activities were more significant for fatty $\mathrm{N}$-aromatic amide, and the one with hydroxyl group (paracetamol) showed the highest antioxidant activity. These in vitro assays indicate that amide derivatives can be utilized as antioxidants in pharmaceutical applications and others such as food supplements.

\section{Acknowledgments}

None.

\section{Conflicts of Interset}

None.

\section{References}

1. Pham-Huy LA, He H, Pham-Huy C. Free Radicals, Antioxidants in Disease and Health. Int J Biomed Sci. 2008;4(2): 89-96.

2. Yumrutas O, Sokmen A, Ozturk N. Determination of in vitro antioxidant activities and phenolic compounds of different extracts of Salvia verticillata ssp. verticillata and spp. amasiaca from Turkey's flora. $J$ Appl Pharm Sci. 2011;1(10):43-46.

3. Brand-Williams W, Cuvelier ME, Berset C. LWT-Food Sci Technol. 2008;28:25-30.

4. Kushwaha N, Saini RK, Kushwaha SKS (2011) Synthesis of some amide derivatives and their biological activity. Int J Chem Tech Res. 1995;3(1):203-209.

5. Saeedi M, Goli F, Mahdavi M, et al. Synthesis and Biological Investigation of some Novel Sulfonamide and Amide Derivatives Containing Coumarin Moieties. Iran J Pharm Res. 2018;13(3):881-892.

6. Huczyński A, Janczak J, Stefańska J, et al. Synthesis and antimicrobial activity of amide derivatives of polyether antibiotic-salinomycin. Bioorg Med Chem Lett. 2012;22(14):4697-4702.

7. Narasimhan B, Belsare D, Pharande D, et al. Esters, amides and substituted derivatives of cinnamic acid: synthesis, antimicrobial activity and QSAR investigations. Eur J Med Chem. 2014;39(10):827-834.

8. Aly AA, Nour-El-Din AM. Functionality of amidines and amidrazones ARKIVOC i, 153-194. Special Issue Reviews and Accounts, 2008. p. 153-194.

9. http://erpub.org/siteadmin/upload/3389ER915010.pdf

10. Narasimhan B, Narang R, Judge V, et al. Synthesis, antimicrobial and QSAR studies of substituted anilides. General Papers. 2011;15:112126.

11. Farshori NN, Ahmad A, Khan AU, et al. A facile, one-pot synthesis, characterization and antimicrobial activity of o-hydroxy anilide derivatives and 1-substituted-1,3-dicyclohexylurea analogs of long chain carboxylic acids. Eur J Med Chem. 2011;46(4):1433-1438.

12. Malki F, Touati A, Hamza K. Antioxidant activity of a series of amides. J Mater Environ Sci. 2016;7(3):936-941.

13. Malki F, Touati A, Rahal S, et al. Total synthesis of monocyclic pyrimidinium betaines with fatty alkyl chain. Asian Chem. 2011;23(3):961-967.
14. Webb N. Org Synth Coll, 1947. p. 182.

15. Malki F, Touati A, Moulay S. et al. Towards Green Synthesis of Fatty Alkanilides. J MaterEnviron Sci. 2007;8(1):238-243.

16. Roginsky V, Lissi EA. Review of methods to determine chain-breaking antioxidant activity in food. Food Chem. 1995;92(2):235-254.

17. Ak T, Gülçin İ. Antioxidant and radical scavenging properties of curcumin. Chem Biol Interact. 2007;174(1):27-37.

18. Gülçin İ, Kireçci E, Akkemik E. Antioxidant, antibacterial, and anticandidal activities of an aquatic plant: duckweed (Lemna minor L. Lemnaceae). Turk J Biol. 2010;34(2):175-188.

19. Blois MS. Antioxidant determinations by the use of stable free radical. Nature. 1958;181:1199-1200.

20. Frankel EN, Meyer AS. The problems of using one-dimensional methods to evaluate multifunctional food and biological antioxidants. J Sci Food Agric. 200;80(13):1925-1940.

21. Gülçin I. Oktay M, Kıreçci E, et al. Screening of antioxidant and antimicrobial activities of anise (Pimpella anisum L.) seed extracts. Food Chem. 2003;83(3):371-382.

22. Shimada K, Fujikawa K, Yahara K, et al. Antioxidative properties of xanthan on the autoxidation of soybean oil in cyclodextrin emulsion. $J$ Agric Food Chem. 1992;40(6):945-948.

23. Elmastaş M, Gülçin I, Işildak Ö, et al. Radical scavenging activity and antioxidant capacity of bay leaf extracts. J Iran Chem Soc. 2006;3(3):258-266.

24. Gülçin I, Elmastat M, Aboul-Enein HY. Determination of Antioxidant and radical scavenging activity of basil (Ocimum basilicum L. Family Lamiaceae) assayed by different methodologies. Phytother Res. 2007;21(4):354-361.

25. Oyaizu M. Studies on products of browning reaction prepared from glucosamine. Jpn J Nut. 1986;44:307-315.

26. Krinsky NI. Antioxidant functions of carotenoids. Free Radic Biol Med. 1989;7(6):617-635.

27. Tepe B, Daferera D, Sokmen A. Antimicrobial and antioxidant activities of the essential oil and various extracts of salvia tomentosa Miller (Lamiaceae). Food Chem. 2005;90(3):333-340.

28. Motlhanka DM, Habtemariam S, Houghton P. Free radical scavenging activity of crude extracts and 4'-OMethyl-(-) epigallocatechin isolated from roots of Cassine transvaalensis from Botswana. Afr J Biomed Res. 2008;11:55-63.

29. Duan XJ, Zhang WW, Li XM. Evaluation of antioxidant property of extract obtained from a red alga. Polysiphonis ureceolta. Food Chem. 2008;95(1):37-43.

30. Wang H, Gao XD, Zhou GC, et al. In Vitro and in Vivo antioxidant activity of aqueous Extract from Choerospondias axillaris fruit. Food Chem. 2008;106(3):888-895.

31. Ferreira D, Slade D, Marais JPJ. Flavans and Proanthocyanidins," in Flavonoids: Chemistry, Biochemistry and Applications. CRC Press, Boca Raton, USA, 2006. p. 553-616. 